\title{
Cobalamin production by Lactobacillus coryniformis: biochemical identification of the synthetized corrinoid and genomic analysis of the biosynthetic cluster
}

\author{
Andrea Carolina Torres, Verónica Vannini, Julieta Bonacina, Graciela Font, Lucila Saavedra and María Pía Taranto*
}

\begin{abstract}
Background: Despite the fact that most vitamins are present in a variety of foods, malnutrition, unbalanced diets or insufficient intake of foods are still the cause of vitamin deficiencies in humans in some countries. Vitamin $B_{12}$ (Cobalamin) is a complex compound that is only naturally produced by bacteria and archea. It has been reported that certain strains belonging to lactic acid bacteria group are capable of synthesized water-soluble vitamins such as those included in the B-group, as vitamin $B_{12}$. In this context, the goal of the present paper was to evaluate and characterize the production of vitamin $B_{12}$ in Lactobacillus coryniformis CRL 1001, a heterofermentative strain isolated from silage.

Results: Cell extract of L. coryniformis CRL 1001, isolated from silage, is able to correct the coenzyme $B_{12}$ requirement of Salmonella enterica serovar Typhimurium AR 2680 in minimal medium. The chemical characterization of the corrinoid-like molecule isolated from CRL 1001 cell extract using HPLC and mass spectrometry is reported. The majority of the corrinoid produced by this strain has adenine like Coa-ligand instead 5,6-dimethylbenzimidazole. Genomic studies revealed the presence of the complete machinery of the anaerobic biosynthesis pathway of coenzyme $B_{12}$. The detected genes encode all proteins for the corrin ring biosynthesis and for the binding of upper ( $\beta$ ) and lower (a) ligands in one continuous stretch of the chromosome.

Conclusions: The results here described show for the first time that L. coryniformis subsp. coryniformis CRL 1001 is able to produce pseudocobalamin containing adenine instead of 5,6-dimethlbenzimidazole in the Coa-ligand. Genomic analysis allowed the identification and characterization of the complete de novo biosynthetic pathway of the corrinoid produced by the CRL 1001 strain.
\end{abstract}

Keywords: Lactobacillus, Cobalamin production, Coenzyme $B_{12}$ gene cluster organization

\section{Background}

Lactic acid bacteria (LAB) are a heterogeneous group of bacteria extensively used as starter cultures for the fermented foods development. Because of their metabolism, this group of bacteria can improve the safety, shelf life, nutritional value, flavour and overall quality of fermented products. Some strains have shown to exert a large range of beneficial properties in humans, and they are frequently used as probiotic microorganisms. In

\footnotetext{
* Correspondence: ptaranto@cerela.org.ar

Centro de Referencia para Lactobacilos (CERELA)-CONICET, San Miguel de Tucumán, Tucumán, Argentina
}

addition, certain LAB strains are able to produce and release compounds with biological activity in foods, sometimes referred as nutraceuticals [1]. These micronutrients are used as a cofactor in numerous enzymatic reactions, as it is the case of vitamin $B_{12}$.

Conceptually, the definition of vitamin $B_{12}$ generally describes a type of cobalt corrinoid, belonging to the cobalamin $(\mathrm{Cbl})$ group. On the other hand, vitamin $\mathrm{B}_{12}$ is the form of the vitamin obtained during process of industrial production but this form not exists in the nature [2]. Naturally, this compound is found as desoxyadenosilcobalamin (coenzyme $\mathrm{B}_{12}$ ), 
methylcobalamin or pseudocobalamin, among other forms. Concerning the structure, the cobalamin molecule present 3 parts main: (i) the central corrinic ring with the four ligands of a cobalt ion, (ii) a superior (or beta) ligand that is attached to adenosyl o methyl group, and (iii) the lower ligand (or alfa), usually dimethylbenzimidazole (DMB). It has been described that in some anaerobic bacteria, the adenine and other ligands can replace DMB giving as a result pseudocobalamin (pseudo- $\mathrm{B}_{12}$ ) and other active cofactors [3]. This soluble compound is one of the most complex non-polymeric macromolecules produced in the cell and only a reduced group of bacteria and archaea are able to synthesize it. The bacteria present in the rumen of cattle, sheep and other ruminants are the responsible of its synthesis. Humans do not have such microbiota in their large intestine and must absorb the coenzyme from natural sources such as meat, fish, eggs, and pharmaceutical products [4]. A diet sufficient in vitamin $\mathrm{B}_{12}$ is essential to prevent severe pathologies (megaloblastic anaemia, pancytopenia, peripheral neuropathy, increased risk of myocardial infarction and stroke, others) some of which are irreversible [5-8]. Thus, the use of vitamin-producer bacteria appears as an appropriate alternative to produce innovative foods with added nutritional value [9]. However, the production of vitamin $B_{12}$ is strain specific and an unusual trait present in certain strains of the Lactobacillus genus. The production of this metabolite by Lactobacillus reuteri strains has been described for the first time by us and confirmed later by others authors [10-13]. Moreover, we validated that the corrinoid

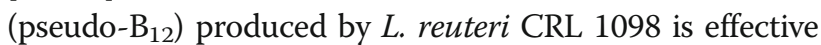
in preventing the hematological and neurological pathologies (anaemia, growth retardation, others) caused by a diet deficiency in vitamin $\mathrm{B}_{12}$ in a combined animal model (pregnant mice and their offspring) [14, 15]. More recently, De Angelis et al. [16] described the production and the genetic organization of the complete de novo biosynthetic pathway of vitamin $\mathrm{B}_{12}$ in Lactobacillus rossiae DSM 15814. Among other lactobacilli, previous evidence suggested that two strains of L. coryniformis (CECT 5711 and strain 394) are capable of producing the antimicrobial compound reuterin $[17,18]$, an enzymatic pathway that involves a cobalamin-dependent enzyme, the glycerol dehydratase [19]. No further analysis related to vitamin $\mathrm{B}_{12}$ production was carried out in those studies. Based on this previous evidence, the purpose of the present study was to evaluate the vitamin $\mathrm{B}_{12}$ production in L. coryniformis CRL 1001, a heterofermentative strain isolated from silage. Firstly, the compound with cobalamin activity was isolated and biochemically characterized. Next, the genome of the CRL 1001 strain was sequenced by whole genome shotgun (WGS) sequencing strategy and the identification of putative biosynthetic operon involved in the synthesis of the corrinoid was performed by in silico analysis. Finally, a detailed comparative study of the cobalamin gene cluster of L. coryniformis CRL 1001 with those of previously known vitamin-producer strains was performed. The results of this research provide the first evidence of vitamin $\mathrm{B}_{12}$ synthesis by a $L$. coryniformis strain.

\section{Results and discussion}

\section{Cobalamin production by L. coryniformis CRL 1001}

In order to evidence the production of cobalamin by $L$. coryniformis CRL 1001, we use $S$. Typhimurium AR 2680 (metE cbiB) [20] as indicator strain, grown in minimal medium without vitamin $\mathrm{B}_{12}$ according the methodology described by Taranto et al. [12]. The results of the bioassay showed that the cobalamin requirements of the indicator strain were corrected when Cell Extracts (CE) of L. coryniformis CRL 1001 were added; the halo diameter obtained was similar to that obtained with a solution of standard cyanocobalamin $(\mathrm{CN}-\mathrm{Cbl})$. On the other hand, no growth was detected when the $\mathrm{CE}$ from Lactobacillus plantarum ATCC 8014 (negative control strain) was used. These results would indicate that $L$. coryniformis CRL 1001 is able to produce compounds with cobalamin activity.

To identify the compound with cobalamin activity produced by $L$. coryniformis CRL 1001, the CE was analysed by RP-HPLC. The UV-DAD spectra data revealed the presence of two main peaks that showed similar characteristics with the spectrum obtained for the peak belonging to the CN-Cbl standard. The Retention Times (RT) of these peaks were slightly lower (23.77 $\mathrm{min}$ and $24.20 \mathrm{~min}$ ) to the RT of standard solution of $\mathrm{CN}-\mathrm{Cbl}$ (24.98 min) (Fig. 1). However, when these peaks were collected and analysed using the bioassay, they showed the same ability of vitamin $B_{12}$ complementation than the $\mathrm{CN}-\mathrm{Cbl}$ standard. For this reason, we continued with the identification of both peaks.

\section{Characterization of the corrinoid produced by $L$. coryniformis CRL 1001}

Corrinoid compounds separated by HPLC were analysed by Liquid chromatography-electrospray ionization/tandem mass spectrometry (LC/ESI-MS/MS). The mass module parameters were fine-tuned and optimized with commercial standard of $\mathrm{CN}-\mathrm{Cbl}$. The transition 678.3 $(\mathrm{m} / \mathrm{z})\left[\mathrm{M}+2 \mathrm{H}^{+}\right]^{++}$to $358.7(\mathrm{~m} / \mathrm{z})$ corresponds to the lower ligand, whose $\mathrm{DMB}$ is the aglycon attached to ribofuranose 3-phosphate whereas the transition 678.3 $(\mathrm{m} / \mathrm{z})\left[\mathrm{M}+2 \mathrm{H}^{+}\right]^{++}$to $146.9(\mathrm{~m} / \mathrm{z})$ corresponds to the base DMB. Data transition for pseudo- $B_{12}$ were estimated and calculated according the bibliography as follows: $672.5(\mathrm{~m} / \mathrm{z})\left[\mathrm{M}+2 \mathrm{H}^{+}\right]^{++}$to $347.8(\mathrm{~m} / \mathrm{z})$; this transition corresponds to the lower ligand where adenine is the aglycon attached to ribofuranose 3-phosphate, 


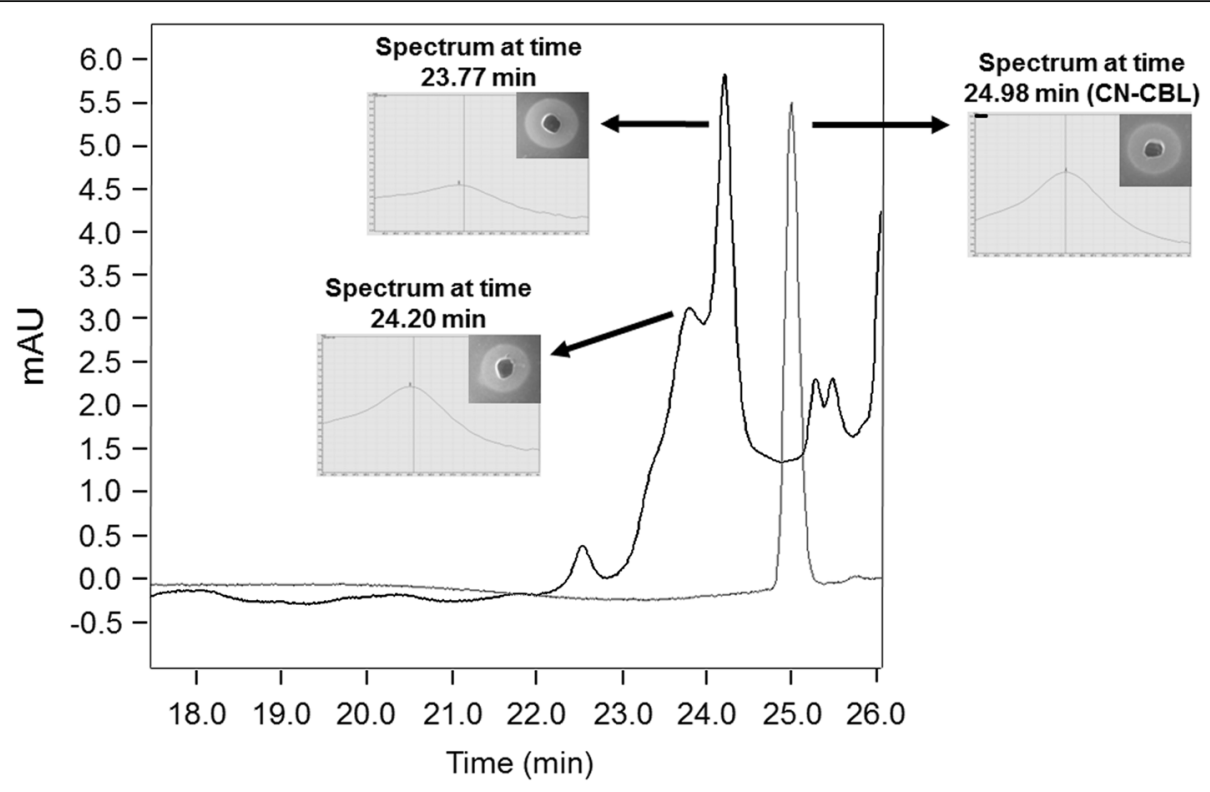

Fig. 1 RP-HPLC chromatogram of the cell extract (CE) from L. coryniformis CRL 1001 (black); CN-Cbl standard (grey). UV-DAD spectrum and bioassay images (annex inset) of CN-Cbl standard and each corrinoid produced by CRL 1001 strain

and the transition $672.5(\mathrm{~m} / \mathrm{z})\left[\mathrm{M}+2 \mathrm{H}^{+}\right]^{++}$to $135.9(\mathrm{~m} / \mathrm{z})$ corresponds to the base adenine [21]. Both transitions were sought in the MS and MS/MS spectra of the peaks with cobalamin activity.

The MS spectra of the active peaks obtained by HPLC indicated that a double charged ion with an approximately $\mathrm{m} / \mathrm{z}$ of $673\left[\mathrm{M}+2 \mathrm{H}^{+}\right]^{++}$was prominent in the two spectra (Fig. 2). The MS/MS spectrum indicated that the dominant ions with a value approximate $\mathrm{m} / \mathrm{z}$ 347.8 were attributable to the substitution in the lower ligand where the adenine is the aglycon attached to ribofuranose 3-phosphate.

The MS showed that the molecular species (corrinoid) synthesized by L. coryniformis CRL 1001 corresponds mainly to Co $\alpha$-[ $\alpha$-(7-adenyl)]-Co $\beta$-cyanocobamide, commonly known as pseudo- $\mathrm{B}_{12}$, an analogue with a structure very similar to vitamin $B_{12}$. In this corrinoid, the DMB moiety is substituted by adenine as lower ligand. This compound is a physiologically important form of the vitamin $B_{12}$ in various microorganisms [22].

The concentration of pseudo- $\mathrm{B}_{12}$ produced by L. coryniformis CRL 1001 was calculated indirectly using a standard curve made with different concentrations of a commercial $\mathrm{CN}-\mathrm{Cbl}$. The area of the peaks obtained in the chromatograms corresponds to approximately $0.94 \mu \mathrm{g} \mathrm{mL}^{-1}$ of pseudo- $\mathrm{B}_{12}$ produced.

\section{Genome identification of vitamin $B_{12}$ cluster in $L$. coryniformis CRL 1001}

The genome sequence of $L$. coryniformis CRL 1001 consisted on 133 contigs with a total size of $2,829,178$ base pairs and an average GC content of $42 \%$. The structural and functional annotation performed with the Rapid Annotations using Subsystems Technology (RAST) server [23] allowed us to identify 3341 coding sequences (CDS) and 82 structural RNAs (58 tRNAs) [23]. Additionally, it was observed that 332 subsystems that include the $44 \%$ of the identified CDS are represented in the chromosome.

In silico genomic analyses revealed the presence of 32 open reading frames (ORFs) related to the coenzyme $B_{12}$ production ( $c b i, c o b$, hem and $c b l$ gene cluster) located immediately adjacent to the $p d u$ operon (22 ORFs) which encodes the enzymes necessary for the use of glycerol or propanediol as carbon source (Fig. 3). As described in other species, the close association of these two gene clusters is probably a reflection of the requirements of cobalamin or its corrinoid derivatives for the use of propanodiol [24]. Almost all predicted genes ( $p d u, c b i, c o b$ and hem) are in the same orientation, except for $p o c R$, which encodes for a transcriptional regulator with $45 \%$ identity and $69 \%$ positive matches at the amino acid level, with its homolog in L. reuteri JCM 1112 [25]. Interestingly, the two clusters ( $p d u$ and cbicob-hem) have a few intergenic regions and their average $\mathrm{G}+\mathrm{C}$ content (52\%) differ from that of the draft genome sequence of L. coryniformis CRL 1001 (42\%). As described by Morita et al. [10] for L. reuteri JCM 1112, this data could explain the existence of a genomic island acquired through horizontal gene transfer.

Among $p d u$ genes, $p d u C, p d u D$ and $p d u E$ were found. These genes encode for the three subunits of the glycerol 


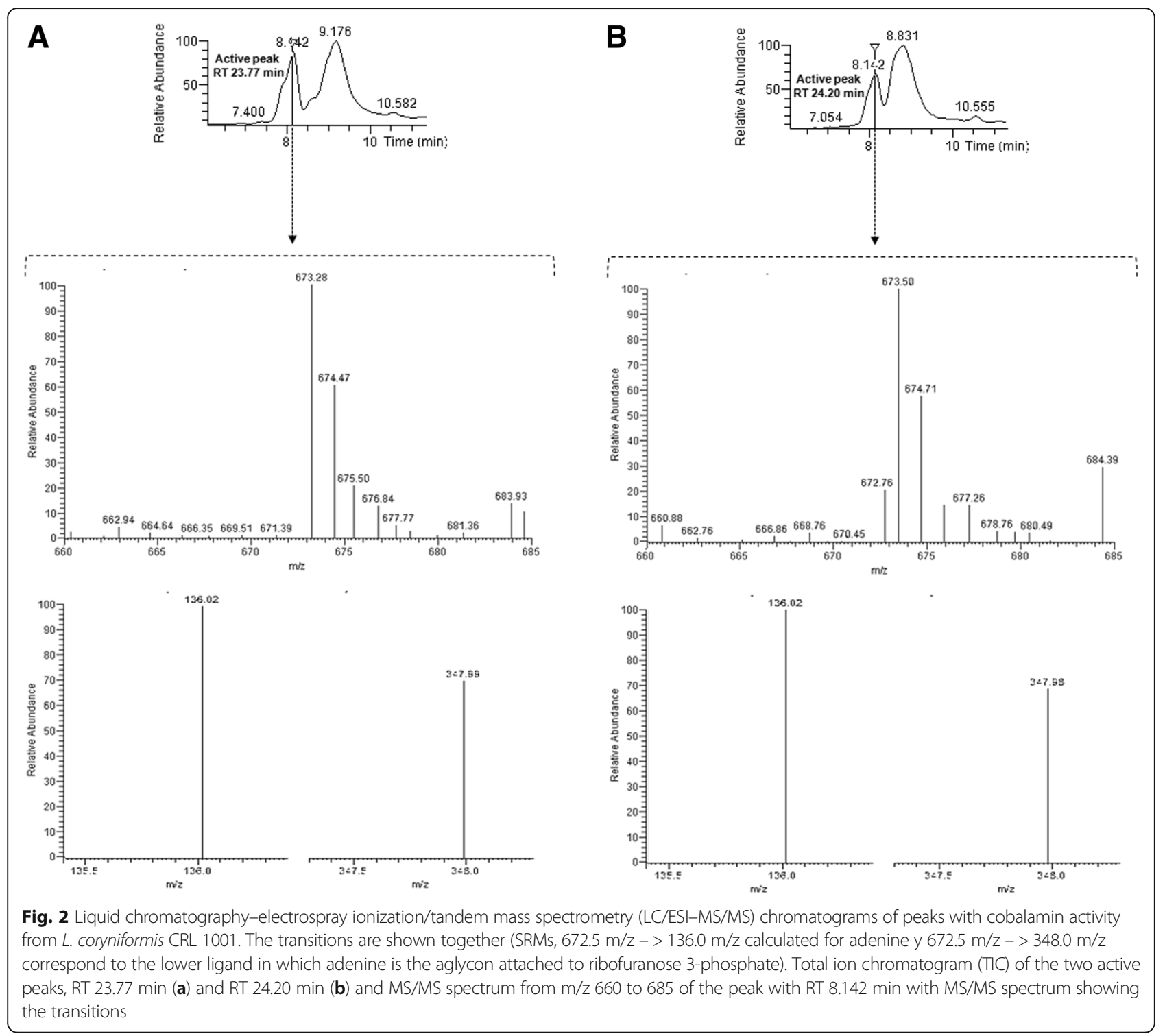

dehydratase, an enzyme that requires cobalamin as a cofactor for performing it catalytic activity [19]. Additionally, a gene coding for the L-threonine kinase PduX, was localized. This protein, described in Salmonella enterica, transfers a phosphoryl group to a free L-threonine and it is involved in de novo synthesis of Adenosyl cobalamin and the assimilation of cobyric acid (Cby) [26].

Downstream of the $p d u$ operon, seventeen $c b i$ genes (cbiA, B, C, D, E, T, F, G, H, J, K, L, M, N, Q, O, and $P$ ) and cys $G$ gene were in silico identified. The proteins encoded by these genes are required in most of the reactions that take place in the cell during the corrinoid ring synthesis of the cobalamin molecule [27].

Regarding $c o b$ genes $\operatorname{cob} A, D, U, S$ and $C$ were found. $\operatorname{cob} A$ encodes an adenosyltransferase, a binding protein of adenosyl group as $\beta$-ligand [28]. The gene $c o b D$ encodes an L-threonine-O-3-phosphate descarboxylase that is involved in the amino-propanol arm synthesis [29] while cob $U, S$ and $C$ encode the proteins that participate in the attachment of the amino-propanol arm and in the assembly of the nucleotide loop that connects the lower cobalt ligand to the corrinoid ring [30].

Finally, downstream of the $c b i$ and $c o b$ genes, five hem genes (hem $A, C, B, L$ and $D$ ) were detected in a similar way as observed for other microorganisms that produce cobalamin anaerobically [31]. The proteins encoded by these genes are involved in the first steps of the cobalamin biosynthesis, the synthesis of uroporphyrinogen III from L-glutamyl-tRNA(glu). At the same time, the hemD/cobA gene, encoding a single polypeptide with both uroporphyrinogen III synthase and methyltransferase activity was detected [32].

Interestingly, the genome of $L$. coryniformis CRL 1001 lacks cobT gene, contrary to that observed in 


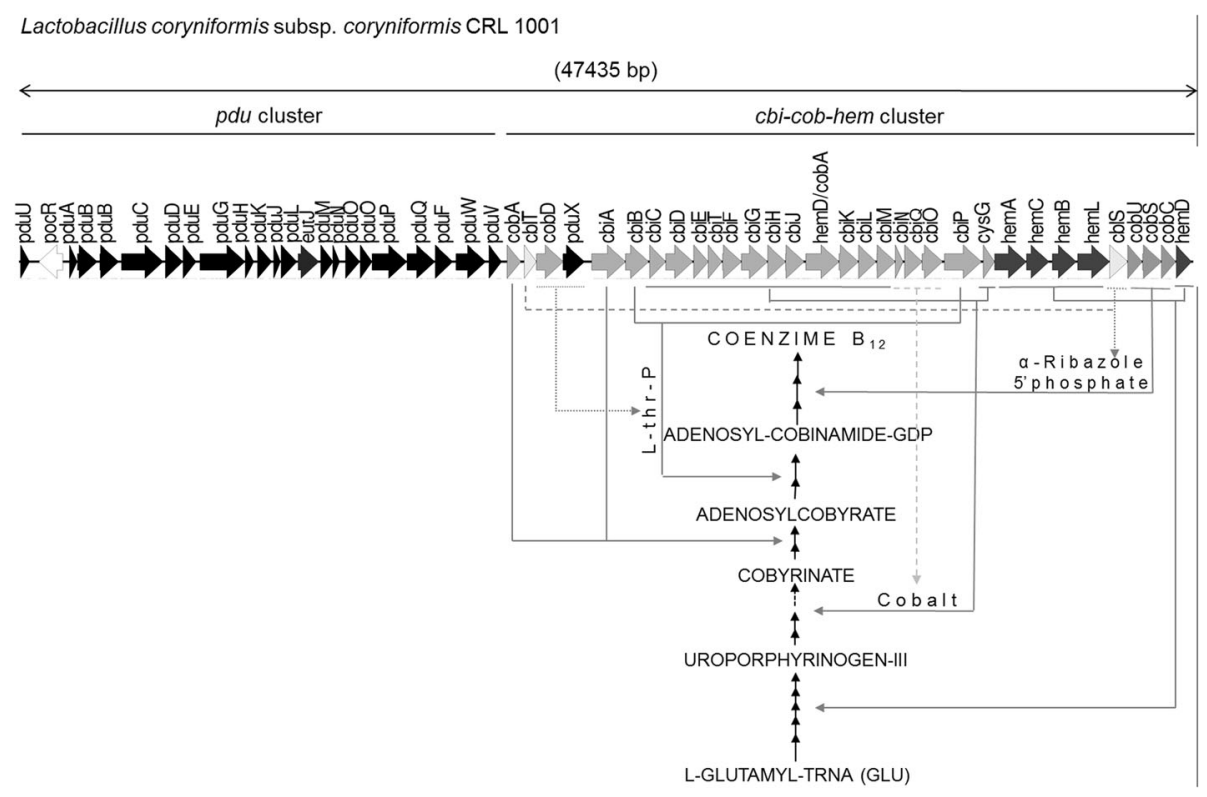

Fig. 3 Schematic representation of the pdu and cbi-cob-hem clusters of L. coryniformis CRL 1001. The arrows represent genes involved in: synthesis of uroporphyrinogen III (dark grey), synthesis of cobinamide, lower ligand and cobalt transport (light grey), propanediol degradation (black) and pocR, a transcriptional regulator (white). On the simplified $B_{12}$ synthesis pathway scheme, genes encoding proteins involved in synthesis of coenzyme (full lines) and synthesis and transport of intermediaries (incomplete lines) are highlighted

the cobalamin biosynthetic cluster of $L$. reuteri CRL 1098 [11]. Instead, two $c b l$ genes were detected, $c b l S$ and $c b l T$, encoding for a $\alpha$-ribazole phosphoribosyl kinase and a $\alpha$-ribazole transporter, respectively. These results might suggest an alternative pathway for $\alpha$-ribazole salvaging and the $\alpha$-ribazole-P synthesis in L. coryniformis CRL 1001, as it is described in Listeria [33].

\section{In silico comparative analysis of the biosynthetic cluster of vitamin $B_{12}$}

The cobalamin biosynthetic gene cluster has been identified in a few Lactobacillus species, including L. reuteri [11] and more recently in $L$. rossiae [16]. Both strains are obligate hetero-fermentative LAB [34]. L. coryniformis CRL 1001 is the first facultative heterofermentative strain described as cobalamin producer.

Comparative genomic studies among L. coryniformis CRL 1001, L. reuteri DSM 20016 and L. rossiae DSM 15814 strains evidenced a conserved genetic organization of the coenzyme $B_{12}$ biosynthetic genes. In these three strains, the cbi-cob-hem cluster is adjacent to the $p d u$ cluster (Fig. 4). However, contrasting other cobalamin producer strains, this two gene sets ( $p d u$ and cbi-cob-hem) have an opposite orientation in the $L$. rossiae genome. Morita et al. [10] suggested that both gene cluster might have been acquired by horizontal transfer and inserted independently in these genomes.
As previously described for cobalamin producer anaerobic strains, the hem genes were located among the $c b i$ cob genes in all the organisms under comparison.

The $c b l S$ and $c b l T$ genes identified in the genome of $L$. coryniformis were also detected in the genome of $L$. rossiae where they have an identical localization in the $c b i$ cob-hem cluster. However, these genes are absent in the genome of $L$. reuteri. The cobT gene, encoding a Nicotinate mononucleotide (NaMN): base phosphoribosyltransferase, has only been described in this latter specie for the strains L. reuteri CRL 1098 (AY780645.1), L. reuteri JCM 1112 (NC_010609.1) and L. reuteri DSM 20016 (NZ_AZDD00000000.1).

Until now, among the Lactobacillus strains described as cobalamin producers, $L$. coryniformis CRL 1001 is the only strain harbouring the gene encoding for PduX on its genome (Fig. 4).

A comparative analysis between the deduced amino acid sequences of each ORF included in the vitamin $B_{12}$ biosynthetic cluster of $L$. coryniformis CRL 1001 and those from L. rossiae, L. reuteri, Listeria sp. and Salmonella sp. using the BLASTP algorithm, was performed. We found that most of L. coryniformis CRL 1001 proteins have the highest percentage of identity with those identified in L. rossiae strain (Table 1), with the exception of CobD and PduX, which were more similar to Listeria proteins. This result indicates that the aminopropanol phosphate synthesis from L-threonine in $L$. coryniformis CRL 1001 is similar to that performed by 


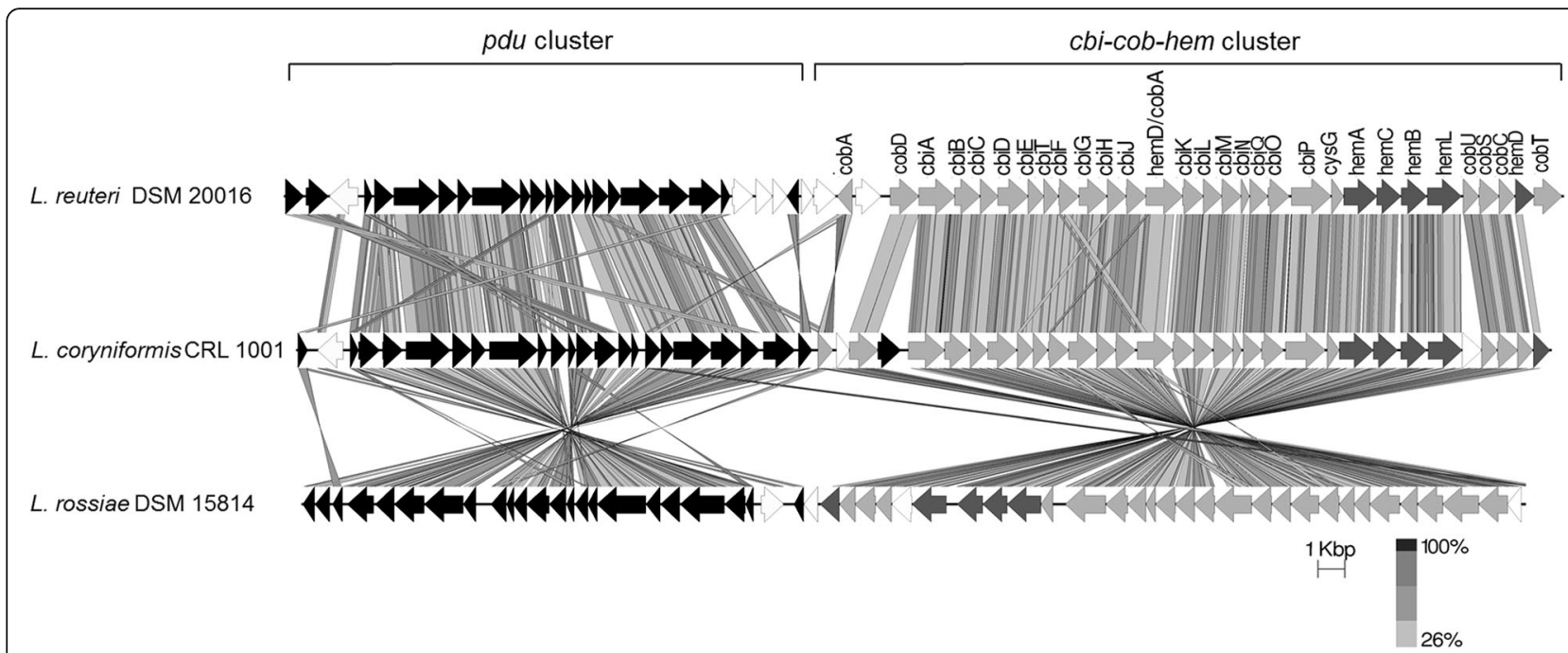

Fig. 4 Linear comparison of the pdu cluster and the cbi-cob-hem cluster between L. coryniformis CRL 1001, L. reuteri DSM 20016 (up) and L. rossie DSM 15814 (down). Genes in the gene clusters are depicted by arrows indicating the transcription direction. Genes conserved between the three genomes are colored in grey and black bars indicating orthologous regions

Listeria strains, previously described by Fan et al. [26]. These results suggest an alternative vitamin $\mathrm{B}_{12}$ biosynthetic pathway in this genus.

\section{Conclusion}

In this work, we demonstrated for the first time that $L$. coryniformis subsp. coryniformis CRL 1001 is able to produce corrinoids with cobalamin activity. The chemical characterization of the molecule isolated from CRL 1001 CE using HPLC and mass spectrometry show that the majority of corrinoid produced is pseudocobalamin that has adenine like Coo-ligand instead 5,6-dimethylbenzimidazole. This molecule is one of the forms of coenzyme $B_{12}$ occurring in nature. Genomic studies revealed the presence of the complete anaerobic biosynthetic pathway of coenzyme $\mathrm{B}_{12}$. The comparative analysis of the cobalamin gene cluster of $L$. coryniformis CRL 1001 with those present in strains previously described as vitamin producers, revealed that this strain bears the genes cblS-cblT only present in the genome of L. rossiae DSM 15814. Moreover, $p d u X$ gene has not been detected in another strain of the Lactobacillus genus. The results of this work provide the first evidence of vitamin $\mathrm{B}_{12}$ synthesis by a $L$. coryniformis strain.

\section{Methods}

\section{Strains, media and culture conditions}

L. coryniformis CRL 1001, a strain originally isolated from silage, belongs to the CERELA-CONICET culture collection. The CRL 1001 strain was grown in ManRogosa-Sharpe (MRS) broth and Vitamin $\mathrm{B}_{12}$ Assay Medium (Merck, Germany) overnight at $37{ }^{\circ} \mathrm{C}$ without shaking. S. Typhimurium AR 2680, a strain with mutations in the genes metE and $c b i B$, was used as indicator strain in bioassays for cobalamin determination. This strain was grown in Luria-Bertani (LB) broth at $37^{\circ} \mathrm{C}$ with aeration. To perform the bioassay, the indicator strain was grown in minimal A medium $(\mathrm{NaCl}$, $0.5 \mathrm{~g} \mathrm{~L}^{-1}, \mathrm{Na}_{2} \mathrm{HPO}_{4}, 6 \mathrm{~g} \mathrm{~L}^{-1} ; \mathrm{KH}_{2} \mathrm{PO}_{4}, 3 \mathrm{~g} \mathrm{~L}^{-1}, \mathrm{NH}_{4} \mathrm{Cl}$ $1 \mathrm{~g} \mathrm{~L}^{-1}$; glucose, $4 \mathrm{~g} \mathrm{~L}^{-1}$; $\mathrm{MgSO}_{4}, 2 \mathrm{mM}, \mathrm{CaCl}_{2} 0.1 \mathrm{mM}$ ). As negative control, L. plantarum ATCC 8014 was employed.

\section{Preparation of cultures and cell-extracts}

The production of corrinoid type-cobalamin by $L$. coryniformis CRL 1001 was detected as following: a culture was inoculated duplicated in vitamin $\mathrm{B}_{12}$-free assay medium, grown at $37{ }^{\circ} \mathrm{C}$ for $16 \mathrm{~h}$ and subcultured three times in the same medium before use. Cell Extracts (CE) were prepared from $100 \mathrm{~mL}$ of culture grown for $24 \mathrm{~h}$; after harvesting $(8000 \times g, 5 \mathrm{~min})$, cells were washed in $10 \mathrm{~mL}$ of $0.1 \mathrm{M}$ phosphate buffer $\mathrm{pH} 7.0$ twice and resuspended in $10 \mathrm{~mL}$ of the same buffer and were fasted overnight at $2{ }^{\circ} \mathrm{C}$. Cells were harvested $(8000 \times g, 5 \mathrm{~min})$ and resuspended in $10 \mathrm{~mL}$ of extraction buffer $(0.1 \mathrm{M}$ $\mathrm{Na}_{2} \mathrm{HPO}_{4}$, pH 4.5 reached with, $\left.0.005 \% \mathrm{KCN}\right)$. The cell suspension obtained was autoclaved at $120{ }^{\circ} \mathrm{C}$ for $20 \mathrm{~min}$, the pellet was separated by centrifugation $(10,000 \times g, 20 \mathrm{~min})$, and the supernatant was passed over a solid-phase extraction (SPE) column C18-E (Phenomenex) with $500 \mathrm{mg}$ particle size and $6 \mathrm{~mL}$ reservoir volume; the column was previously activated with $6 \mathrm{~mL}$ of methanol HPLC degree (Loba Chemie, India). The column was washed with 2 volumes of ultra-purified water twice to remove salts and other hydrophilic contaminants. The potentials corrinoid was eluted with 1 
Table 1 Comparative analysis of the deduced amino acid sequences of each ORF involved in vitamin $B_{12}$ biosynthesis cluster of Lactobacillus coryniformis CRL 1001 with those present in other cobalamin producer strains

\begin{tabular}{|c|c|c|c|c|c|c|c|c|c|}
\hline \multicolumn{2}{|c|}{ L. coryniformis CRL 1001} & \multicolumn{2}{|c|}{ Lactobacillus rossiae } & \multicolumn{2}{|c|}{ Lactobacillus reuteri } & \multicolumn{2}{|l|}{ Listeria sp. } & \multicolumn{2}{|c|}{ Salmonella sp. } \\
\hline Protein & Length (aa) & Length (aa) & Identity (\%) & Length (aa) & Identity (\%) & Length (aa) & Identity (\%) & Length (aa) & Identity (\%) \\
\hline CobA & 188 & 189 & 71 & 194 & 68 & 188 & 56 & 176 & 40 \\
\hline $\mathrm{Cb} 1 \mathrm{~T}$ & 166 & 174 & 61 & $-*$ & $-*$ & 164 & 54 & 192 & 36 \\
\hline Cob D & 360 & 364 & 41 & 362 & 42 & 361 & 45 & 364 & 42 \\
\hline Pdu X & 288 & $-*$ & $-*$ & $-^{*}$ & $-*$ & 291 & 37 & 288 & 36 \\
\hline Cbi A & 456 & 457 & 59 & 454 & 61 & 452 & 54 & 459 & 50 \\
\hline Cbi B & 312 & 319 & 62 & 319 & 58 & 316 & 55 & 459 & 50 \\
\hline Cbi C & 217 & 213 & 68 & 228 & 64 & 210 & 64 & 210 & 51 \\
\hline Cbi D & 365 & 375 & 74 & 383 & 73 & 373 & 55 & 370 & 50 \\
\hline Cbi E & 199 & 200 & 54 & 199 & 55 & 198 & 53 & 201 & 43 \\
\hline Cbi T & 184 & 188 & 56 & 184 & 63 & 189 & 53 & 192 & 44 \\
\hline Cbi F & 254 & 258 & 83 & 253 & 77 & 249 & 76 & 257 & 71 \\
\hline Cbi G & 351 & 355 & 55 & 351 & 53 & 343 & 46 & 351 & 36 \\
\hline Cbi H & 241 & 241 & 77 & 241 & 78 & 241 & 67 & 241 & 58 \\
\hline Cbi J & 249 & 250 & 47 & 252 & 56 & 250 & 45 & 263 & 33 \\
\hline Hem D/Cob A & 449 & 468 & 45 & 464 & 48 & 493 & 40 & $-*$ & $-{ }^{*}$ \\
\hline Cbi K & 262 & 259 & 47 & 259 & 53 & 261 & 49 & 264 & 46 \\
\hline Cbi L & 235 & 232 & 47 & 232 & 62 & 236 & 48 & 237 & 38 \\
\hline Cbi M & 248 & 239 & 77 & 247 & 74 & 244 & 59 & 245 & 57 \\
\hline Cbi N & 104 & 114 & 76 & 103 & 63 & 82 & 56 & 93 & 45 \\
\hline Cbi Q & 235 & 230 & 54 & 225 & 56 & 225 & 37 & 225 & 34 \\
\hline Cbi O & 276 & 274 & 65 & 269 & 63 & 268 & 54 & 271 & 51 \\
\hline Cbi P & 499 & 499 & 67 & 503 & 70 & 511 & 57 & 506 & 56 \\
\hline Cys G & 153 & 148 & 45 & 152 & 51 & 146 & 44 & 154 & 32 \\
\hline Hem A & 429 & 431 & 53 & 421 & 55 & - & - & - & - \\
\hline Hem C & 305 & 309 & 55 & 305 & 55 & - & - & - & - \\
\hline Hem B & 324 & 321 & 81 & 323 & 77 & - & - & - & - \\
\hline Hem L & 430 & 430 & 77 & 431 & 75 & - & - & - & - \\
\hline $\mathrm{Cbl} \mathrm{S}$ & 242 & 252 & 53 & $-*$ & $-*$ & 252 & 38 & 273 & 37 \\
\hline Cob U & 196 & 197 & 46 & 196 & 54 & 185 & 41 & 181 & 40 \\
\hline Cob S & 253 & 257 & 52 & 253 & 53 & 248 & 38 & 244 & 35 \\
\hline Cob C & 194 & 194 & 46 & 196 & 43 & 191 & 32 & 202 & 29 \\
\hline Hem D & 209 & 243 & 46 & 236 & 49 & - & - & - & - \\
\hline
\end{tabular}

Length variation among deduced aminoacid sequences of each ORF related to cobalamin production in Lactobacillus, Salmonella and Listeria strains. The percentage of protein sequence identity is related to those detected in Lactobacillus coryniformis CRL1001. (-*) ORF not detected. (-) ORF not detected among cbi-cob-hem cluster

volume of methanol and concentrated to dryness in vacuum at $30{ }^{\circ} \mathrm{C}$. The residue was dissolved in $100 \mu \mathrm{l}$ of $50 \%$ methanol and stored in the dark at $-20^{\circ} \mathrm{C}$ until use.

\section{Cobalamin detection}

To demonstrate the production of cobalamin by L. coryniformis CRL 1001, a bioassay using $S$. Typhimurium AR 2680 as indicator strain was performed. The CE from $L$. coryniformis CRL 1001 was examined for its ability to correct the cobalamin requirement of the AR 2680 strain in minimal medium. For this purpose, $S$. Typhimurium AR 2680 cells were grown for 16 to $18 \mathrm{~h}$ in LB medium; the active culture was seeded onto minimal agar medium specific for this strain. Four wells were performed in each agar plate. Fifteen microliters of $\mathrm{CE}$ from L. coryniformis CRL 1001 (dilutions $11^{-1}$ and $11^{-1}$ ) were loaded in each well and incubated $24 \mathrm{~h}$ under anaerobic conditions at $37{ }^{\circ} \mathrm{C}$. As comparative standard of 
vitamin $\mathrm{B}_{12}$, a commercial cianocobalamin $(\mathrm{CN}-\mathrm{Cbl})$ solution $\left(0.5 \mu \mathrm{g} \mathrm{mL}^{-1}\right)$ (Sigma-Aldrich, cod. V2876) was used. The CE of $L$. plantarum ATCC 8014, obtained in a similar way that the CE of L. coryniformis CRL 1001, was used as negative control.

\section{Isolation and characterization of the corrinoid}

To isolate the corrinoid produced by $L$. coryniformis CRL 1001, the CE was loaded onto a $\mathrm{C}_{18}$ solid-phase $5 \mu \mathrm{m}$ extraction column $(250 \times 4.6 \mathrm{~mm})$ (Shimadzu Shim-Pack VP-ODS) and purified by reverse-phase (RP)HPLC using a LC_20 AT system automated gradient controller with a SIL-20 ${ }^{\text {a }} \mathrm{HT}$ autosampler and a SPD-M20A diode array UV-visible detector at $361 \mathrm{~nm}$ (Shimadzu Corporation, Kyoto, Japan). The column was thermostated at $25{ }^{\circ} \mathrm{C}$. The mobile phases was filtered through a $0.22 \mu \mathrm{m}$ membrane filter and vacuum degassed prior to use. The mobile phases were prepared with ultra-purified water with $0.1 \%$ formic acid (Solvent A) and methanol with $0.1 \%(\mathrm{v} / \mathrm{v})$ formic acid (Solvent B).

The protocol used was described by Yu et al. [35] with some modifications. The following mobile phase gradients (Solvent B) were used: 0-2 min (20\%); 2-5 min (35\%); 5-22 $\min$ (100\%); 22-26 min (100\%); 26-36 min (20\%); and 36-45 $\min (5 \%)$; this step was followed by the final passage of Solvent B at $5 \%$ for re-optimization of the parameters in the module HPLC. The volume of injection was $100 \mu \mathrm{l}$. The HPLC was operated in a constant flow mode and the flow rate was kept at $0.5 \mathrm{ml} \mathrm{min}{ }^{-1}$.

The peaks with RT close to the RT of CN-Cbl standard (Sigma-Aldrich, cod. V2876) (concentration $=5 \mu \mathrm{g} \mathrm{mL}^{-1}$ ) were collected and concentrated to dryness under vacuum at $30{ }^{\circ} \mathrm{C}$. Then, the peaks were dissolved with methanol $50 \%$ and stored in the dark at $-20{ }^{\circ} \mathrm{C}$ until use. The vitamin $B_{12}$ activity of each collected peak at $1.10^{-1}$ and $1.100^{-1}$ dilutions was detected using the bioassay with the Salmonella strain. Peaks with vitamin $\mathrm{B}_{12}$ activity positive were analysed by mass spectrometry.

The active peaks collected from the RP-HPLC were analysed using Ultimate 3000 RSLC Dionex - Thermo Scientic with a $\mathrm{C}_{18}$ column $50 \times 2.1 \mathrm{~mm}$ that contained 1.9 micron particles (Hypersil-GOLD). The solvents for Liquid Chromatography were solvent A (ultra-purified water with $0.1 \%$ formic acid) and solvent B (methanol with $0.1 \%$ formic acid). The flow rate was $0.2 \mathrm{~mL} \mathrm{~min}^{-1}$ and the injection volume was $10 \mu \mathrm{L}$. Chromatography was in gradient, and the solvent composition was chosen such that the compound of interest eluted 8-9 min after injection. The mobile phase gradients (solvent B) were maintained during a $35 \mathrm{~min}$ run: $0-2 \mathrm{~min}(5 \%) ; 2-5 \mathrm{~min}$ (35\%); 5-22 min (100\%); 22-24 min (100\%); 24-28 min (5\%); and $28-35 \mathrm{~min}(5 \%)$, this step was followed by $50 \%$ of solvent B for re-optimization of the parameters in the module HPLC coupled with Mass. A single peak for each sample was eluted and the area of the signal was used for quantitative analysis. Positive-ion MS/MS experiments were performed in product mode on a triple quadrupole mass spectrometer (Thermo Scientific TSQ Quantum Access Max).

\section{Genome sequencing}

The genomic DNA was extracted from the cultured bacterium according to Pospiech and Neuman [36] and the genome sequence was determined using a wholegenome shotgun (WGS) strategy with an Ion Torrent personal genome machine (Life Technologies). Quality filtered reads were in silico assembled via the DNASTAR NGen assembler by MR DNA (Shallowater, TX).

This Whole Genome Shotgun project has been deposited at DDBJ/EMBL/GenBank under the accession LNUL00000000. The version described in this paper is LNUL01000000.

\section{Genomic analysis}

The $L$. coryniformis CRL 1001 genome sequence analysis was carried out following different approaches. Open Reading Frame (ORF) prediction and functional assignment was automatically performed using the RAST webserver (http://rast.nmpdr.org/), ORF Finder (http:// www.ncbi.nlm.nih.gov/gorf/gorf.html) and BLASTX (http://blast.ncbi.nlm.nih.gov/Blast.cgi). The classification of the ORFs was carried out using BLASTP alignment against the database (http://blast.ncbi.nlm.nih.gov/ Blast.cgi), using a cut-off E-value of $1.10^{-4}$ with at least $30 \%$ of identity across a minimum of $50 \%$ of a given protein length.

\section{Comparative analyses}

BLAST-based comparative analyses were performed of each one of the CDS of the cbi-cob-hem cluster between L. coryniformis CRL 1001 and two well-characterized Lactobacillus strains described as corrinoids compound producers with cobalamin activity, L. reuteri DSM 20016 and $L$. rossiae DSM 15814 . This analysis was performed employing Easyfig 2.1: a genome comparison visualizer application [37].

\section{Acknowledgements \\ We thank Dr. Gastón Pourrieux (Centro de Referencia para Lactobacilos, CERELA-CONICET, Argentina) for the technical support with HPLC and spectrometry studies.}

\section{Funding}

This study was carried out with the financial support from CONICET (PIP0406/12) and MinCyT (PICT2011 N0175) from Argentina.

Availability of data and materials

Whole Genome Shotgun project of Lactobacillus coryniformis CRL 1001 has been deposited at DDBJ/EMBL/GenBank under the accession LNUL00000000. The version described in this paper is LNUL01000000. 


\section{Authors' contributions}

ACT carried out biochemical and molecular genetic studies, participated in the drafted the manuscript. MW participated physiological and genetic studies. JB participated in the genomic and comparative analysis. GF participated in the discussion of the study and performed the statistical analysis. LS participated in the design, discussion and coordination of this study and drafting the manuscript. MPT carried out the coordination of this study and participated in the design, discussion and drafting the manuscript. All authors read and approved the final manuscript.

\section{Competing interests}

The authors declare that they have no competing interests.

\section{Consent for publication}

Not applicable.

\section{Ethics approval and consent to participate}

Not applicable.

\section{Received: 2 April 2016 Accepted: 27 September 2016} Published online: 13 October 2016

\section{References}

1. Wildman REC, Wildman R, Wallace TC. Handbook of Nutraceuticals and Functional Foods. 2nd ed. 2006

2. Rucker RB, Suttie JW, McCormick DB. Handbook of Vitamins. 3rd ed. 2001.

3. Martens $J$ H, Barg H, Warren MJ, Jahn D. Microbial production of vitamin $B_{12}$. Appl Microbiol Biotechnol. 2002;58(3):275-85.

4. Herbert V. Prooxidant effects of antioxidant vitamins. Introduction. J Nutr. 1996;126(4 Suppl):1197S-200.

5. Derin S, Koseoglu S, Sahin C, Sahan M. Effect of vitamin $B_{12}$ deficiency on olfactory function. Int Forum Allergy Rhinol. 2016;6:1051-55.

6. Kocaoglu C, Akin F, Caksen H, Boke SB, Arslan S, Aygun S. Cerebral atrophy in a vitamin $B_{12}$-deficient infant of a vegetarian mother. J Health Popul Nutr. 2014;32(2):367-71.

7. von Schenck U, Bender-Gotze C, Koletzko B. Persistence of neurological damage induced by dietary vitamin B-12 deficiency in infancy. Arch Dis Child. 1997;77(2):137-9.

8. Issac TG, Soundarya S, Christopher R, Chandra SR. Vitamin $B_{12}$ deficiency: an important reversible co-morbidity in neuropsychiatric manifestations. Indian J Psychol Med. 2015;37(1):26-9.

9. Hugenholtz J, Smid EJ. Nutraceutical production with food-grade microorganisms. Curr Opin Biotechnol. 2002;13(5):497-507.

10. Morita H, Toh H, Fukuda S, Horikawa H, Oshima K, Suzuki T, Murakami M, Hisamatsu S, Kato Y, Takizawa T, et al. Comparative genome analysis of Lactobacillus reuteri and Lactobacillus fermentum reveal a genomic island for reuterin and cobalamin production. DNA Res. 2008;15(3):151-61.

11. Santos F, Vera JL, van der Heijden R, Valdez G, de Vos WM, Sesma F, Hugenholtz J. The complete coenzyme $\mathrm{B}_{12}$ biosynthesis gene cluster of Lactobacillus reuteri CRL 1098. Microbiology. 2008;154(Pt 1):81-93.

12. Taranto MP, Vera JL, Hugenholtz J, De Valdez GF, Sesma F. Lactobacillus reuteri CRL 1098 produces cobalamin. J Bacteriol. 2003;185(18):5643-7.

13. Sriramulu DD, Liang M, Hernandez-Romero D, Raux-Deery E, Lunsdorf $H$, Parsons JB, Warren MJ, Prentice MB. Lactobacillus reuteri DSM 20016 produces cobalamin-dependent diol dehydratase in metabolosomes and metabolizes 1,2-propanediol by disproportionation. J Bacteriol. 2008;190(13):4559-67.

14. Molina VC, Medici M, Taranto MP, Font de Valdez G. Lactobacillus reuteri CRL 1098 prevents side effects produced by a nutritional vitamin B deficiency. J Appl Microbiol. 2009;106(2):467-73.

15. Molina V, Medici M, Font de Valdez G, Taranto MP. Soybean-based functional food with vitamin $B_{12}$-producing lactic acid bacteria. J Funct Foods. 2012:4(4):831-6.

16. De Angelis M, Bottacini F, Fosso B, Kelleher $P$, Calasso M, Di Cagno R, Ventura M, Picardi E, van Sinderen D, Gobbetti M. Lactobacillus rossiae, a vitamin $B_{12}$ producer, represents a metabolically versatile species within the Genus Lactobacillus. PLoS One. 2014;9(9):e107232.

17. Martin R, Olivares M, Marin ML, Xaus J, Fernandez L, Rodriguez JM. Characterization of a reuterin-producing Lactobacillus coryniformis strain isolated from a goat's milk cheese. Int J Food Microbiol. 2005;104(3):267-77.
18. Tanaka O, Komatsu T, Oshibe A, Cai Y, Miyazaki S, Nakanishi K. Production of 3-hydroxypropionaldehyde in silage inoculated with Lactobacillus coryniformis plus glycerol. Biosci Biotechnol Biochem. 2009;73(7):1494-9.

19. Daniel R, Bobik TA, Gottschalk G. Biochemistry of coenzyme $B_{12}$-dependent glycerol and diol dehydratases and organization of the encoding genes. FEMS Microbiol Rev. 1998;22(5):553-66.

20. Raux E, Lanois A, Levillayer F, Warren MJ, Brody E, Rambach A, Thermes C. Salmonella typhimurium cobalamin (vitamin $\mathrm{B}_{12}$ ) biosynthetic genes: functional studies in S. typhimurium and Escherichia coli. J Bacteriol. 1996; 178(3):753-67.

21. Hashimoto E, Yabuta Y, Takenaka S, Yamaguchi Y, Takenaka H, Watanabe F. Characterization of corrinoid compounds from edible cyanobacterium Nostochopsis sp. J Nutr Sci Vitaminol. 2012;58(1):50-3.

22. Taga ME, Walker GC. Pseudo- $B_{12}$ joins the cofactor family. J Bacteriol. 2008; 190(4):1157-9.

23. Aziz RK, Bartels D, Best AA, DeJongh M, Disz T, Edwards RA, Formsma K, Gerdes S, Glass EM, Kubal M, et al. The RAST Server: rapid annotations using subsystems technology. BMC Genomics. 2008;9:75

24. Bobik TA, Havemann GD, Busch RJ, Williams DS, Aldrich HC. The propanediol utilization (pdu) operon of Salmonella enterica serovar Typhimurium LT2 includes genes necessary for formation of polyhedral organelles involved in coenzyme B(12)-dependent 1, 2-propanediol degradation. J Bacteriol. 1999;181(19):5967-75

25. Santos F, Spinler JK, Saulnier DM, Molenaar D, Teusink B, de Vos WM, Versalovic J, Hugenholtz J. Functional identification in Lactobacillus reuteri of a PocR-like transcription factor regulating glycerol utilization and vitamin $\mathrm{B}_{12}$ synthesis. Microb Cell Fact. 2011;10:55.

26. Fan C, Bobik TA. The PduX enzyme of Salmonella enterica is an L-threonine kinase used for coenzyme $B_{12}$ synthesis. J Biol Chem. 2008;283(17):11322-9.

27. Raux E, Lanois A, Rambach A, Warren MJ, Thermes C. Cobalamin (vitamin B12) biosynthesis: functional characterization of the Bacillus megaterium cbi genes required to convert uroporphyrinogen III into cobyrinic acid a, c-diamide. Biochem J. 1998:335(Pt 1):167-73.

28. Suh S, Escalante-Semerena JC. Purification and initial characterization of the ATP:corrinoid adenosyltransferase encoded by the cobA gene of Salmonella typhimurium. J Bacteriol. 1995;177(4):921-5.

29. Brushaber KR, OToole GA, Escalante-Semerena JC. CobD, a novel enzyme with L-threonine-O-3-phosphate decarboxylase activity, is responsible for the synthesis of (R)-1-amino-2-propanol O-2-phosphate, a proposed new intermediate in cobalamin biosynthesis in Salmonella typhimurium LT2. J Biol Chem. 1998:273(5):2684-91.

30. Maggio-Hall LA, Escalante-Semerena JC. In vitro synthesis of the nucleotide loop of cobalamin by Salmonella typhimurium enzymes. Proc Natl Acad Sci U S A. 1999:96(21):11798-803.

31. Johansson P, Hederstedt L. Organization of genes for tetrapyrrole biosynthesis in gram-positive bacteria. Microbiology. 1999;145(Pt 3):529-38.

32. Vannini V, Rodriguez A, Vera JL, de Valdez GF, Taranto MP, Sesma F. Cloning and heterologous expression of Lactobacillus reuteri uroporphyrinogen III synthase/methyltransferase gene (cobA/hemD): preliminary characterization. Biotechnol Lett. 2011;33(8):1625-32.

33. Gray MJ, Escalante-Semerena JC. A new pathway for the synthesis of alpha-ribazole-phosphate in Listeria innocua. Mol Microbiol. 2010;77(6): 1429-38.

34. De Vos P, Garrity GM, Jones D, Krieg NR, Ludwig W, Rainey FA, Schleifer K, Whitman WB. The firmicutes. In: Parte AC, editor. Bergey's manual of systematic bacteriology second edition, vol. Three. 2nd ed. New York: Springer Science + Business Media; 2009.

35. Yu Y, Zhu X, Shen Y, Yao H, Wang P, Ye K, Wang X, Gu Q. Enhancing the vitamin $B_{12}$ production and growth of Propionibacterium freudenreichii in tofu wastewater via a light-induced vitamin $B_{12}$ riboswitch. Appl Microbiol Biotechnol. 2015:99(24):10481-8.

36. Pospiech A, Neumann B. A versatile quick-prep of genomic DNA from gram-positive bacteria. Trends Genet. 1995;11(6):217-8.

37. Sullivan MJ, Petty NK, Beatson SA. Easyfig: a genome comparison visualizer. Bioinformatics. 2011:27(7):1009-10. 